\title{
Extracellular miRNAs as Biomarkers in Cancer
}

\author{
Jennifer F. Barger ${ }^{*}$, , Mohammad A. Rahman ${ }^{1}$, Devine Jackson ${ }^{1}$, Mario Acunzo², and S. \\ Patrick Nana-Sinkam ${ }^{1,2,3}$ \\ ${ }^{1}$ Division of Pulmonary, Allergy, Critical Care and Sleep Medicine, The Ohio State University, \\ Columbus $\mathrm{OH} 43210$ \\ 2Department of Molecular Virology, Immunology and Medical Genetics, The Ohio State University, \\ Columbus $\mathrm{OH} 43210$ \\ ${ }^{3}$ Division of Medical Oncology, James Comprehensive Cancer Center, The Ohio State University, \\ Columbus $\mathrm{OH} 43210$
}

\section{Introduction}

Cancer is the leading cause of death worldwide, accounting for about 1 in 8 deaths worldwide. According the World Health Organization (WHO), there were 14.1 million new cases and 8.2 million cancer deaths in 2012. The number of cases diagnosed is expected to rise to nearly 20 million cases by $2025 .{ }^{1}$ This creates a global social and economic burden with estimates suggesting that the global cost of cancer is $\$ 2.5$ trillion a year, a combination of healthcare costs, disability and premature death. ${ }^{2}$

Despite significant progress in the battle against cancer, poor patient outcomes are still attributable to several subtypes of cancer. The application of high throughput genomics to tumors has led to the increasing recognition that many cancers are heterogeneous, thus requiring specific molecularly targeted interventions. Investigators have leveraged targeting somatic, epigenetics (methylation) and more recently immunotherapy all in the hopes of curbing mortality from cancers. ${ }^{3-5}$ Approximately 20 years ago, investigators first determined that additional components of the human genome, previously considered to be nonfunctional, are essential to cancer biology. ${ }^{6}$ These small noncoding RNAs termed microRNAs (miRNAs) are often localized to fragile chromosomal regions and have now been implicated in the regulation of the majority of the hallmarks of cancer biology. ${ }^{7}$ In 2008, Hunter and colleagues first determined that miRNAs were detectable in circulation within lipid-encapsulated microvesicles. ${ }^{8}$ The recent discovery of extracellular vesicles (EVs), membrane bound nano-vesicles secreted by cells and detectable in circulation has prompted considerable interest in the field of biomarkers. One particular area of intense focus has been the development of noninvasive biomarkers that may be utilized to better

*Corresponding Author: Jennifer F Barger, Jennifer.Fleming@ osumc.edu, 450 Biomedical Research Tower, 440 W $12^{\text {th }}$ Ave, Columbus OH 43210.

Publisher's Disclaimer: This is a PDF file of an unedited manuscript that has been accepted for publication. As a service to our customers we are providing this early version of the manuscript. The manuscript will undergo copyediting, typesetting, and review of the resulting proof before it is published in its final citable form. Please note that during the production process errors may be discovered which could affect the content, and all legal disclaimers that apply to the journal pertain. 
inform clinical decision-making in cancer diagnosis and therapeutics. Unfortunately, many non-invasive studies have been limited by lack of reproducibility and an unclear understanding regarding the compartment specificity of genetic material in circulation. The EV cargo, mostly nucleotides and proteins, is protected from degradation, reflects the pathogenesis of the cell of origin and can be transferred to recipient cells altering their function making them ideal candidates for novel cancer biomarkers. This review will focus on the growing field of EVs and their contents, specifically microRNAs, in biomarker discovery and the challenges of translating their biology to clinical application.

\section{Extracellular miRNAs in Cancer}

Since their initial discovery in 1993, microRNAs (miRNAs) have been implicated in all steps of tumorigenesis from tumor initiation to metastasis. ${ }^{7}$ miRNAs are small non-coding RNAs that regulate gene expression by targeting mRNA for degradation or translational repression, controlling normal and abnormal physiological processes. ${ }^{9}$ The seminal discovery of miRNAs in serum of patients with B-cell lymphoma by Lawrie and colleagues suggested for the first time the clinical potential of circulating miRNAs for diagnostic or prognostic non-invasive biomarkers. ${ }^{10}$ Subsequently, others have discovered miRNAs in body fluids including saliva, urine and sputum. Circulating miRNAs provide a unique look into the body as their composition and concentration reflect the cell of origin, indicate the presence of viruses and correlate with pathological conditions. ${ }^{11}$

MiRNAs present in biological fluids are protected from RNases by two distinct mechanisms, either packaged into extracellular vesicles or bound in a protein complex composed of Argonaute (AGO) family members. ${ }^{12,13,14}$ While the majority of miRNAs are found complexed with AGO and not in EVs, it is still unclear as to their source and function. ${ }^{15}$ One hypothesis is that the vesicle-free miRNAs are from apoptotic or necrotic cells and thus the clinical relevance of these miRNAs is understated. ${ }^{16}$ Because of hemolysis during the processing of samples, additional AGO-miRNAs are released contaminating blood plasma with irrelevant miRNAs complicating the analysis and clinical utility of vesicle-free miRNAs. In contrast, the packaging of miRNAs into EVs reduces the issues associated with processing of the samples. There is no evidence that these AGO-miRNAs can transfer the miRNA to recipient cells whereas EVs shuttle miRNA to other cells to modulate the gene expression and function of the recipient cells. ${ }^{17}$ While circulating miRNAs are abundant in biological fluids, many questions remain as to their source and function and thus, biomarker research has turned to EVs as a stable and reliable source of functional miRNAs.

\section{EV Biogenesis}

EVs are small membrane-bound nano-vesicles that can be further subdivided based on their size into exosomes (20-150nm), microvesicles (100nm-1um) and apoptotic bodies (1-5um). Additionally, they are sometimes divided based on content, cellular or tissue of origin and the mechanism of release. For example, EVs derived from prostate cancer cells are often referred to as prostastosomes. ${ }^{18}$ The major emphasis on EV biological functions and clinical applications has centered on exosomes and small microvesicles $(<500 \mathrm{~nm})$. Standard methods of isolation including, ultracentrifugation, precipitation or bead-based sorting are 
unable to distinguish between these two populations as they overlap in size and surface marker expression.

Originally, EVs were thought to bud directly from the plasma membrane. However, in the 1980s two groups studying reticulocyte maturation described a more complex mode of EV secretion. They showed that small vesicles were formed by inward budding inside an intracellular endosome, leading to the formation of a multivesicular body (MVB), which could then fuse with the plasma membrane and release its internal vesicles. ${ }^{19}$ In 1987, the word 'exosomes' was proposed for these EVs of endosomal origin. ${ }^{20}$ The existence of this unusual EV secretion pathway was confirmed afterwards in antigen-presenting cells, ${ }^{21,22}$ epithelial cells ${ }^{23}$ and tumor cells. ${ }^{24}$ Several molecules are involved in the biogenesis of EVs, such as the ESCRT machinery, lipids (such as ceramide) and the tetraspanins. However, recent studies suggest that ESCRT-independent mechanisms may be responsible for EV biogenesis as mammalian cells depleted for key ESCRT components still form MVBs. ${ }^{25}$ It is still unknown whether these mechanisms act simultaneously on the same MVB population or on different MVBs. Therefore, alterations in these pathways could affect MVB dynamics and the subsequent release of subpopulations of EVs.

EVs are secreted by all cell types and contain proteins, mRNA, and microRNAs (miRNAs) protected by a lipid bilayer. EVs have also been detected in a number of human body fluids, including plasma, ${ }^{10}$ urine, ${ }^{26}$ breast milk,,${ }^{27}$ cerebrospinal fluid, ${ }^{28}$ malignant ascites ${ }^{29}$ and bronchoalveolar lavage fluid ${ }^{30}$ suggesting their importance in vivo. Cells secrete distinct populations of EVs with unique size, protein and RNA composition, and that these EVs have different effects on recipient cells. ${ }^{31}$ Since mRNA have also been detected in plasma membrane derived microvesicles, ${ }^{32}$ it is currently believed that cells use EVs as a means of extracellular communication and exchange of proteins, lipids and nucleic acids. ${ }^{33}$ Whether subpopulations of EVs display different or identical functions is still unclear.

Due to multiple extracellular and intracellular physiological processes that can be attributed to EVs, there is a need to define biochemical mechanisms that mediate secretion and uptake of these vesicles. It has been shown that diseases like cancer increase the release of EVs. It is becoming increasingly clear that factors that impose stress on cells can mediate the secretion of exosomes. ${ }^{34}$ For example, stress conditions including hypoxia, $\gamma$-irradiation, heparanase, low $\mathrm{pH}$ and cellular detachment induce the release of EVs from cells. Breast cancer cells when exposed to hypoxia release higher numbers of EVs. ${ }^{35} \gamma$-Irradiation influences the production of EVs and this effect is dose and time dependent. ${ }^{36}$ Heparanase is an endoglycosidase that cleaves heparan sulfate and is up-regulated in many cancers. It has been reported that heparanase causes a marked increase in the secretion of EVs. Heparanase not only increases secretion but changes the EV protein composition. ${ }^{37}$ Moreover, the addition of EVs secreted by cells expressing high levels of heparanase altered the behavior of both tumor and host cells. It has been reported that low $\mathrm{pH}$, a hallmark of malignant tumors, plays a key role in aberrantly regulating EV traffic within the tumor mass. In particular, an acidic tumor microenvironment regulates EV mediated malignant tumor cell functions, such as cannibalism and drug resistance. ${ }^{38,39}$ The detachment of cells is a powerful trigger for the release of EVs, which are then concentrated on the surfaces of detached spherical cells. This release is likely to be a novel mechanism for shuttling 
adhesion receptors and possibly other signaling molecules to the cell surface. ${ }^{34}$ Others have shown that cellular activation can alter the dynamics of EV release by increasing the release of specific populations of vesicles. ${ }^{40}$ The fate of MVBs can be either fusion with lysosomes or fusion with the plasma membrane, allowing the release of their contents to the extracellular milieu. Several RAB proteins (RAB11, RAB27 and RAB35) have been shown to be involved in the transport of MVBs to the plasma membrane and in EV secretion. ${ }^{41}$ (Figure 1)

EVs transport miRNAs, as well as proteins and other nucleotides, throughout the body and transfer them to recipient cells in neighboring or distant sites. After delivery, EV miRNAs play functional roles by suppressing expression of target genes or act as ligands to activate immune cells. ${ }^{42}$ Analysis of the stoichiometry of miRNAs and EVs indicate that most individual exosomes do not carry biologically significant numbers of miRNA copies. ${ }^{43}$ This is significant given the increasing number of reports showing functional transfer of miRNAs via EVs and considering that EV transfer of miRNA is a highly selective and infrequent event. ${ }^{44,45,46}$ These findings may point towards the presence of miRNA-rich subpopulations of EVs. Work performed by Palma et al. demonstrated differential packaging of miRNAs and subsequent release of distinct subpopulations of EVs by cancerous cells as compared to normal cells. Increased release of one of the subpopulations possibly resulted from subendocytic changes. ${ }^{47}$

The differences in composition and concentration of miRNAs create disease and cellspecific molecular signatures that can serve as biomarkers for diagnosis, prognosis and therapeutic response. For example, the presence of miR-21 in numerous biological fluids including serum, urine and saliva has both diagnostic and prognostic value in breast, brain, pancreatic, colorectal, lung, gastric and ovarian cancers. ${ }^{48}$ Unfortunately, a lack of consistency between studies and a lack of reproducibility prohibit the translation of many findings into clinical practice. Factors such as insufficient cohorts, technical variations of the source, preparation and purification of the sample and the technological platform for miRNA profiling all contribute to this lack of reproducibility between studies. The enhanced stability and reduced complexity of EV miRNA signatures offer an attractive alternative to vesicle free miRNAs for biomarker discovery. Wang et al. identified miR-21 isolated from the serum EVs as a more sensitive biomarker for hepatocellular carcinoma (HCC) than EVdepleted serum or whole serum. ${ }^{49}$ Corroborating this enrichment of miRNAs in the EVs to serve as biomarkers in HCC, the EV miRNAs were better than circulating miRNAs at distinguishing $\mathrm{HCC}$ from chronic hepatitis B or liver cirrhosis patients. ${ }^{50}$ However, it should be noted that both of these studies contain a small cohort of patients and further validation on larger cohorts needs to be done in order to translate these findings into clinical practice.

\section{EV miRNAs for Clinical Application}

Advances in EV isolation and concentration techniques together with the recognition that EVs contain valuable information regarding disease and pathological conditions has stimulated research on the utility of EVs as noninvasive diagnostic and prognostic tools (Table 1). Screening EV miRNAs holds the potential to not only provide novel non-invasive tools but to provide critical information on aberrant signaling pathways involved in tumor 
initiation, metastasis and therapeutic response that can be translated into novel therapeutic targets.

\section{Diagnostic miRNAs}

Accumulating evidence demonstrates that the molecular constituents of EVs, especially miRNAs hold great promise as novel biomarkers for clinical diagnosis of cancer. EVs found in biological fluids serve as a noninvasive tool to evaluate pathological conditions as their contents reflect that of the cell of origin and the physiological condition. While there are certainly challenges to translating EV biomarkers into the clinic as will be discussed later in this review, there are a number of recent studies that show the promise of EV miRNA signatures. EV miRNA biomarkers appear suited to serve as diagnostics for early detection and to distinguish between benign and malignant disease. The symptoms of cancer often mirror those of other diseases making it difficult to diagnose without further invasive tests. Liquid biopsies, offer a non-invasive alternative to the standard diagnostic test, the invasive tissue biopsy. Histological examination of tissue via biopsy is invasive, expensive and provides only a single snapshot of the heterogeneity of the tumor and the surrounding microenvironment leaving many details behind. EV miRNAs enriched in body fluids that can distinguish between malignant or benign tumors would reduce additional invasive tests and unnecessary surgeries for patients with benign tumors or non-cancer conditions.

The clinical characteristics of pancreatic cancer are similar to those of chronic pancreatitis or solid pseudopapillary tumor and current markers, carcinoembryonic antigen (CEA) and CA 19-9 exhibit low sensitivity and specificity. Serum EV miR-17-5p and miR-21 levels were significantly elevated in patients with pancreatic cancer compared to non-prostate cancer patients. ${ }^{51}$ The diagnostic value of miR-21 and miR-17-5p was determined by ROC curve analyses and was capable of discriminating PC from non-PC. Using cutoff values of 7.693 and 6.826 resulted in specificities and sensitivities of $72.7 \%$ and $92.6 \%$ for miR-17-5p and 95.5\% and $81.5 \%$ for miR-21, respectively. ${ }^{51} \mathrm{EV}$ miR-21 is also elevated in a number of other cancer types indicating it may be a significant regulator of tumorigenesis. ${ }^{48}$ Because it is elevated in numerous cancers, it should be used in combination with other markers to distinguish the cancer's origin or other clinical features to establish a clinical diagnosis. Similar to pancreatic cancer, due to the lack of early symptoms and a lack of early detection methods, ovarian cancer is often detected in the late stage with few therapeutic options available and poor 5-year survival rate..$^{52}$ Meng and colleagues screened a cohort of 163 ovarian cancer patients, 20 patients with benign ovarian diseases and 32 healthy women for the expression a panel of six miRNAs (miR-141, miR-373, miR-200a, miR-200b, miR-200c and miR-429) in serum derived EVs. Since miR-141 and miR-429 were not detectable in the serum EVs of these patients, they were excluded from further assessment. This group determined that miR-200 family members miR-200a, miR-200b and miR-200c expression levels could distinguish malignant from benign ovarian tumors with a sensitivity of $88 \%$ and specificity of $90 \%$. Additionally, this study determined that elevated miR-200b and miR-200c levels correlated with serum CA125 levels, the current standard of care for diagnosis, monitoring treatment response and predicting prognosis. ${ }^{53}$ These studies illustrate the potential of EV miRNAs to be used as a screen for early detection or to differentiate between benign and malignant disease. 


\section{Prognostic and Predictive miRNA}

Because of the accumulating evidence that EV miRNAs influence tumor behavior and alter the microenvironment, they represent excellent candidates as prognostic biomarkers. While the majority of this data is in cancer cell lines and mouse models, a few studies demonstrate the potential of translating the in vitro analysis into the clinic. A recent study by Zhang et al. showed that astrocyte derived EV miR-19a reduced PTEN expression to promote outgrowth of metastatic tumor cells in the brain. ${ }^{54} \mathrm{~A}$ recent study identified a panel of miRNAs whose expression is higher in metastatic sporadic melanoma compared to familial melanoma patients, but found no significant differences in miRNA expression between the familial patients and unaffected controls. ${ }^{55}$ The miR-17-92a cluster is upregulated by genetic amplification in colorectal cancer, however only EV miR-19a is an independent risk factor for overall survival and disease-free survival. ${ }^{56}$ In pancreatic cancer, levels of miR-17-5p correlate with advanced stage and metastasis. ${ }^{51}$

Current methods for monitoring tumor recurrence or response to therapy are invasive or nonexistent. EV miRNA signatures have the potential to provide sequential and serial evaluation of response to therapies enabling a more dynamic approach to personalizing a therapeutic strategy. While preliminary, a recent study identified EV miR-1290 and miR-375 as promising prognostic markers for castrate resistant prostate cancer. ${ }^{57} \mathrm{In}$ an effort to predict toxicities associated with radiation therapy, Dinh et al evaluated EV miRNAs from lung cancer patients treated with radiation and although the cohort was small, they identified changes in miR-150 and miR-29a that correlate with a risk for radiation therapy toxicity. ${ }^{58}$ Comparison of EV miRNAs from HCC patients undergoing liver transplant identified miR-718 expression as a predictor of recurrent disease. ${ }^{59}$

Taken together, these studies illustrate the tremendous potential of EV miRNAs to determine the prognosis and predict therapeutic response and recurrence.

\section{Therapeutics}

Increasing evidence demonstrates the role for EVs in intercellular communication influencing cellular processes in tumor initiation, progression and metastasis. Thus, in addition to serving as biomarkers, EVs are possible novel anti-cancer targets. While this research is still in its infancy, studies have begun to identify key proteins involved in the biogenesis and release including ESCRT and Rab27a. Preliminary results suggest that targeting the biogenesis pathway may have therapeutic significance, reducing tumor growth and metastasis via reduction of exosome production or release. Using a melanoma mouse model, Peinado et al. showed that depletion of Rab27a lowered circulating EVs and reduced primary tumor growth and metastasis. ${ }^{60}$ However, because EVs are released almost ubiquitously by all cell types, a major challenge for translating this approach to clinical application is to specifically target tumor EVs without disrupting normal cell function. An alternative therapeutic approach is to remove tumor specific EVs from circulation. ${ }^{61}$ This application is limited to the identification of specific markers such as HER-2 expressing EVs in HER-2 overexpressing breast cancer to identify this tumor specific population. ${ }^{62}$ In this case, HER-2 expressing EVs interfere with therapy and are associated with relapse and aggression. ${ }^{63}$ Thus, depletion of the HER-2 expressing EVs from circulation may improve 
clinical outcome. Whether or not selecting for HER-2 positive EVs can deplete sufficient tumor specific EVs to achieve a durable clinical response remains to be tested.

In addition to exploring the therapeutic efficacy of targeting tumor EVs, research is expanding to exploit the clinical utility of EVs to deliver miRNAs as a form of personalized therapeutics or to enhance therapeutic response. EVs are natural, small and malleable allowing them to cross biological membranes. ${ }^{64}$ The lipid bilayer protects the contents and EVs are easy to manipulate. Small molecules, proteins or RNAs can be loaded into EVs via transfection or electroporation. ${ }^{65}$ Current approaches towards EV therapeutics for cancer include using immunostimulated EVs derived from a patient's monocytes in order to activate the immune system to recognize and attack the tumor cells and as a vehicle for delivery of therapeutics including tumor suppressive miRNAs or anti-miRNAs to tumors. ${ }^{66,67}$ Like other approaches, the use of EVs to deliver specific miRNAs or miRNA inhibitors is limited by biodistribution and off-target effects. ${ }^{67}$ Unmodified EVs administered in animal models accumulate in the liver, kidney and spleen. Specific targeting can be achieved for tumors that express specific receptors (HER-2 or EGFR) by incorporating the ligands or homing peptides into the EV membrane. ${ }^{63,68}$ However, incorporating these artificial proteins may interfere with the expression, trafficking or membrane stability of the EV. More research is needed to identify novel targeting mechanisms to achieve specificity and efficient targeting of EVs to tumors. In addition, current methods of isolating EVs are time consuming and expensive. Further work is needed to scale up isolation methods while maintaining purity in order to translate EVs as a therapeutic into the clinic.

The search for cancer biomarkers in various tumor EVs is under intense investigation both in vitro and in the clinic. Potential use of some of these biomarkers, as tools for diagnosis/ prognosis and therapy of many cancers, is a new hopeful thread in cancer research. However, there is still a long way before reaching the promising goal. Multiple clinical trials evaluating the diagnostic, prognostic and therapeutic potential of EVs (Table 2).

\section{Future of EV miRNA in clinical application}

Tissue biopsies remain the gold standard for molecular evaluation of cancer, however this is invasive and limited sampling presents a number of clinical challenges. Tissues are heterogeneous and a biopsy only gives a small sample of the entire tumor, thus potentially missing valuable clinical information. Additionally, it is difficult to obtain multiple samples that reflect the evolution of the tumor over time and in response to therapy. For certain malignancies tissue is not obtainable due to the location and accessibility. These challenges highlight the immediate need for better methods for early detection, diagnosis and therapeutic monitoring. There is intense research underway to evaluate specific molecular signatures of EV content, especially miRNAs to replace the invasive tissue biopsy with a liquid biopsy.

Liquid biopsies are obtained primarily through blood and allow for early detection of disease and serial sampling during disease progression and treatment. ${ }^{69}$ However, the identification and validation of clinical biomarkers for disease and treatment response are impeded by the complexity of biological fluid samples. EVs eliminate these constraints. EVs contain tissue- 
specific signatures and are enriched with cargo that is selectively packaged to reflect the pathological condition of its cell of origin. Analyzing the contents of EVs obtained from body fluids reduces the complexity of the sample while retaining important biological components that can serve as biomarkers.

While there are numerous advantages to using liquid biopsies and more specifically EV miRNA signatures for biomarkers, there are several challenges that currently limit the clinical applicability. Current methods for EV isolation include ultracentrifugation, size exclusion or the use of precipitating reagents. ${ }^{70}$ Ultracentrifugation, the most commonly used method in the lab is time consuming and requires a larger volume of sample making this impractical for high throughput clinical applications. This method also requires expensive and specific equipment that may not be available on a widespread platform to translate into the clinical lab. Precipitation reagents also pull down protein aggregates that complicate downstream analysis and may mask disease-specific markers. Similar to ultracentrifugation, size exclusion columns separate the EVs based on size. This method is compatible with a small sample size and does not require expensive equipment, making it potentially useful for clinical use. However, there is mounting evidence that there is heterogeneity within EVs separated by size. Proteomic and miRNA signatures distinguish subpopulations of EVs separated by surface markers. ${ }^{31,40,71,72}$ Much more research is needed to determine whether these populations are truly unique and carry out distinct functions or serve as reliable biomarkers.

Novel strategies for isolation and enrichment are needed to fully harness the clinical potential of EVs. Alternative methods are actively being researched including methods that utilize size and EV surface marker expression to select for exosomes, including microfluidic chips, magnetic beads and flow cytometry based separation. ${ }^{73}$ While these methods have great promise, there is still a great deal of investigation required to fully understand the specific population of EVs that these methods are selecting for and whether these methods will yield sufficient quantity and quality of EVs for downstream applications. ${ }^{74}$

Following isolation and concentration of EVs, there is a lack of consistency in downstream applications to assess their content. There are multiple methods to detect miRNAs in EVs including qPCR, microarray and next generation sequencing all of which have different sensitivities of detection. These are technical challenges that contribute to the inconsistency of miRNA signatures across multiple platforms. This is further complicated by a lack of standards for normalizing techniques and isolation methods. Further, clinical features including sex, age, race and diurnal variation and processing of the sample also alter the composition and concentration of miRNAs and thus must be more robustly studied to personalize signatures based on these features.

The promise of EV miRNA signatures as biomarkers for cancer is evident. EVs are abundant in body fluids including blood, miRNA integrity is maintained in the EV and EV miRNA fingerprints mirror the tissue and physiologic condition. These features make them ideal candidates for biomarkers. There are a number of studies demonstrating the potential clinical utility of EV miRNA signatures, however their translation to the clinic is impeded by technical hurdles to isolate and enrich EVs and a lack of standardized methods for analysis. 


\section{Supplementary Material}

Refer to Web version on PubMed Central for supplementary material.

\section{Acknowledgments}

This work was supported by NIH T32HL007946 (J.F.B), NCI 1R01CA190740-01A1 (S.P.N.), NIH

1RO1DA040395-01 (S.P.N) and Worly Lung Cancer Early Detection Fund (S.P.N)

\section{References}

1. Ferlay J, Soerjomataram I, Dikshit R, et al. Cancer incidence and mortality worldwide: Sources, methods and major patterns in GLOBOCAN 2012. Int J Cancer. 2015; 136(5):E359-86. [PubMed: 25220842]

2. Lopes, G. Investing in cancer prevention and control to reduce global economic burden. ASCO Connection Web site; http://connection.asco.org/commentary. Updated 2016. Accessed 05/01, 2016

3. Dancey JE, Bedard PL, Onetto N, Hudson TJ. The genetic basis for cancer treatment decisions. Cell. 2012; 148(3):409-420. [PubMed: 22304912]

4. Brien GL, Valerio DG, Armstrong SA. Exploiting the epigenome to control cancer-promoting geneexpression programs. Cancer Cell. 2016; 29(4):464-476. [PubMed: 27070701]

5. Mandal R, Chan TA. Personalized oncology meets immunology: The path toward precision immunotherapy. Cancer Discov. 2016

6. Lagos-Quintana M, Rauhut R, Lendeckel W, Tuschl T. Identification of novel genes coding for small expressed RNAs. Science. 2001; 294(5543):853-858. [PubMed: 11679670]

7. Pichler M, Calin GA. MicroRNAs in cancer: From developmental genes in worms to their clinical application in patients. Br J Cancer. 2015; 113(4):569-573. [PubMed: 26158421]

8. Hunter MP, Ismail $\mathrm{N}$, Zhang $\mathrm{X}$, et al. Detection of microRNA expression in human peripheral blood microvesicles. PLoS One. 2008; 3(11):e3694. [PubMed: 19002258]

9. Bartel DP. MicroRNAs: Genomics, biogenesis, mechanism, and function. Cell. 2004; 116(2):281297. [PubMed: 14744438]

10. Lawrie CH, Gal S, Dunlop HM, et al. Detection of elevated levels of tumour-associated microRNAs in serum of patients with diffuse large B-cell lymphoma. Br J Haematol. 2008; 141(5):672-675. [PubMed: 18318758]

11. Larrea E, Sole C, Manterola L, et al. New concepts in cancer biomarkers: Circulating miRNAs in liquid biopsies. Int J Mol Sci. 2016; 17(5)doi: 10.3390/ijms17050627

12. Turchinovich A, Burwinkel B. Distinct AGO1 and AGO2 associated miRNA profiles in human cells and blood plasma. RNA Biol. 2012; 9(8):1066-1075. [PubMed: 22858679]

13. Turchinovich A, Weiz L, Langheinz A, Burwinkel B. Characterization of extracellular circulating microRNA. Nucleic Acids Res. 2011; 39(16):7223-7233. [PubMed: 21609964]

14. Arroyo JD, Chevillet JR, Kroh EM, et al. Argonaute2 complexes carry a population of circulating microRNAs independent of vesicles in human plasma. Proc Natl Acad Sci U S A. 2011; 108(12): 5003-5008. [PubMed: 21383194]

15. Turchinovich A, Tonevitsky AG, Cho WC, Burwinkel B. Check and mate to exosomal extracellular miRNA: New lesson from a new approach. Front Mol Biosci. 2015; 2:11. [PubMed: 25988178]

16. Turchinovich A, Weiz L, Burwinkel B. Extracellular miRNAs: The mystery of their origin and function. Trends Biochem Sci. 2012; 37(11):460-465. [PubMed: 22944280]

17. Valadi H, Ekstrom K, Bossios A, Sjostrand M, Lee JJ, Lotvall JO. Exosome-mediated transfer of mRNAs and microRNAs is a novel mechanism of genetic exchange between cells. Nat Cell Biol. 2007; 9(6):654-659. [PubMed: 17486113]

18. Aalberts M, Stout TA, Stoorvogel W. Prostasomes: Extracellular vesicles from the prostate. Reproduction. 2013; 147(1):R1-14. [PubMed: 24149515] 
19. Pan BT, Teng K, Wu C, Adam M, Johnstone RM. Electron microscopic evidence for externalization of the transferrin receptor in vesicular form in sheep reticulocytes. J Cell Biol. 1985; 101(3):942-948. [PubMed: 2993317]

20. Johnstone RM, Adam M, Hammond JR, Orr L, Turbide C. Vesicle formation during reticulocyte maturation. association of plasma membrane activities with released vesicles (exosomes). J Biol Chem. 1987; 262(19):9412-9420. [PubMed: 3597417]

21. Zitvogel L, Regnault A, Lozier A, et al. Eradication of established murine tumors using a novel cell-free vaccine: Dendritic cell-derived exosomes. Nat Med. 1998; 4(5):594-600. [PubMed: 9585234]

22. Raposo G, Nijman HW, Stoorvogel W, et al. B lymphocytes secrete antigen-presenting vesicles. J Exp Med. 1996; 183(3):1161-1172. [PubMed: 8642258]

23. van Niel G, Raposo G, Candalh C, et al. Intestinal epithelial cells secrete exosome-like vesicles. Gastroenterology. 2001; 121(2):337-349. [PubMed: 11487543]

24. Wolfers J, Lozier A, Raposo G, et al. Tumor-derived exosomes are a source of shared tumor rejection antigens for CTL cross-priming. Nat Med. 2001; 7(3):297-303. [PubMed: 11231627]

25. Stuffers S, Sem Wegner C, Stenmark H, Brech A. Multivesicular endosome biogenesis in the absence of ESCRTs. Traffic. 2009; 10(7):925-937. [PubMed: 19490536]

26. Pisitkun T, Shen RF, Knepper MA. Identification and proteomic profiling of exosomes in human urine. Proc Natl Acad Sci U S A. 2004; 101(36):13368-13373. [PubMed: 15326289]

27. Admyre C, Johansson SM, Qazi KR, et al. Exosomes with immune modulatory features are present in human breast milk. J Immunol. 2007; 179(3):1969-1978. [PubMed: 17641064]

28. Street JM, Barran PE, Mackay CL, et al. Identification and proteomic profiling of exosomes in human cerebrospinal fluid. J Transl Med. 2012; 10 5-5876-10-5.

29. Andre F, Schartz NE, Movassagh M, et al. Malignant effusions and immunogenic tumour-derived exosomes. Lancet. 2002; 360(9329):295-305. [PubMed: 12147373]

30. Admyre C, Grunewald J, Thyberg J, et al. Exosomes with major histocompatibility complex class II and costimulatory molecules are present in human BAL fluid. Eur Respir J. 2003; 22(4):578583. [PubMed: 14582906]

31. Willms E, Johansson HJ, Mager I, et al. Cells release subpopulations of exosomes with distinct molecular and biological properties. Sci Rep. 2016; 6:22519. [PubMed: 26931825]

32. Ratajczak J, Miekus K, Kucia M, et al. Embryonic stem cell-derived microvesicles reprogram hematopoietic progenitors: Evidence for horizontal transfer of mRNA and protein delivery. Leukemia. 2006; 20(5):847-856. [PubMed: 16453000]

33. Raposo G, Stoorvogel W. Extracellular vesicles: Exosomes, microvesicles, and friends. J Cell Biol. 2013; 200(4):373-383. [PubMed: 23420871]

34. Koumangoye RB, Sakwe AM, Goodwin JS, Patel T, Ochieng J. Detachment of breast tumor cells induces rapid secretion of exosomes which subsequently mediate cellular adhesion and spreading. PLoS One. 2011; 6(9):e24234. [PubMed: 21915303]

35. King HW, Michael MZ, Gleadle JM. Hypoxic enhancement of exosome release by breast cancer cells. BMC Cancer. 2012; 12 421-2407-12-421.

36. Arscott WT, Tandle AT, Zhao S, et al. Ionizing radiation and glioblastoma exosomes: Implications in tumor biology and cell migration. Transl Oncol. 2013; 6(6):638-648. [PubMed: 24466366]

37. Thompson CA, Purushothaman A, Ramani VC, Vlodavsky I, Sanderson RD. Heparanase regulates secretion, composition, and function of tumor cell-derived exosomes. J Biol Chem. 2013; 288(14): 10093-10099. [PubMed: 23430739]

38. De Milito A, Iessi E, Logozzi M, et al. Proton pump inhibitors induce apoptosis of human B-cell tumors through a caspase-independent mechanism involving reactive oxygen species. Cancer Res. 2007; 67(11):5408-5417. [PubMed: 17545622]

39. Lugini L, Matarrese P, Tinari A, et al. Cannibalism of live lymphocytes by human metastatic but not primary melanoma cells. Cancer Res. 2006; 66(7):3629-3638. [PubMed: 16585188]

40. Bobrie A, Colombo M, Krumeich S, Raposo G, Thery C. Diverse subpopulations of vesicles secreted by different intracellular mechanisms are present in exosome preparations obtained by differential ultracentrifugation. J Extracell Vesicles. 2012; 1 eCollection 2012. doi: 10.3402/ jev.v1i0.18397 
41. Kowal J, Tkach M, Thery C. Biogenesis and secretion of exosomes. Curr Opin Cell Biol. 2014; 29:116-125. [PubMed: 24959705]

42. Ekstrom K, Valadi H, Sjostrand M, et al. Characterization of mRNA and microRNA in human mast cell-derived exosomes and their transfer to other mast cells and blood CD34 progenitor cells. J Extracell Vesicles. 2012; 1 eCollection 2012. doi: 10.3402/jev.v1i0.18389

43. Chevillet JR, Kang Q, Ruf IK, et al. Quantitative and stoichiometric analysis of the microRNA content of exosomes. Proc Natl Acad Sci U S A. 2014; 111(41):14888-14893. [PubMed: 25267620]

44. Squadrito ML, Baer C, Burdet F, et al. Endogenous RNAs modulate microRNA sorting to exosomes and transfer to acceptor cells. Cell Rep. 2014; 8(5):1432-1446. [PubMed: 25159140]

45. Pegtel DM, Cosmopoulos K, Thorley-Lawson DA, et al. Functional delivery of viral miRNAs via exosomes. Proc Natl Acad Sci U S A. 2010; 107(14):6328-6333. [PubMed: 20304794]

46. Alexander M, Hu R, Runtsch MC, et al. Exosome-delivered microRNAs modulate the inflammatory response to endotoxin. Nat Commun. 2015; 6:7321. [PubMed: 26084661]

47. Palma J, Yaddanapudi SC, Pigati L, et al. MicroRNAs are exported from malignant cells in customized particles. Nucleic Acids Res. 2012; 40(18):9125-9138. [PubMed: 22772984]

48. Shi J. Considering exosomal miR-21 as a biomarker for cancer. J Clin Med. 2016; 5(4)doi: $10.3390 / \mathrm{jcm} 5040042$

49. Wang H, Hou L, Li A, Duan Y, Gao H, Song X. Expression of serum exosomal microRNA-21 in human hepatocellular carcinoma. Biomed Res Int. 2014; 2014:864894. [PubMed: 24963487]

50. Sohn W, Kim J, Kang SH, et al. Serum exosomal microRNAs as novel biomarkers for hepatocellular carcinoma. Exp Mol Med. 2015; 47:e184. [PubMed: 26380927]

51. Que R, Ding G, Chen J, Cao L. Analysis of serum exosomal microRNAs and clinicopathologic features of patients with pancreatic adenocarcinoma. World J Surg Oncol. 2013; 11 219-7819-11-219.

52. Jayson GC, Kohn EC, Kitchener HC, Ledermann JA. Ovarian cancer. Lancet. 2014; 384(9951): 1376-1388. [PubMed: 24767708]

53. Meng X, Muller V, Milde-Langosch K, Trillsch F, Pantel K, Schwarzenbach H. Diagnostic and prognostic relevance of circulating exosomal miR-373, miR-200a, miR-200b and miR-200c in patients with epithelial ovarian cancer. Oncotarget. 2016

54. Zhang L, Zhang S, Yao J, et al. Microenvironment-induced PTEN loss by exosomal microRNA primes brain metastasis outgrowth. Nature. 2015; 527(7576):100-104. [PubMed: 26479035]

55. Pfeffer SR, Grossmann KF, Cassidy PB, et al. Detection of exosomal miRNAs in the plasma of melanoma patients. J Clin Med. 2015; 4(12):2012-2027. [PubMed: 26694476]

56. Matsumura T, Sugimachi K, Iinuma H, et al. Exosomal microRNA in serum is a novel biomarker of recurrence in human colorectal cancer. Br J Cancer. 2015; 113(2):275-281. [PubMed: 26057451]

57. Huang X, Yuan T, Liang M, et al. Exosomal miR-1290 and miR-375 as prognostic markers in castration-resistant prostate cancer. Eur Urol. 2015; 67(1):33-41. [PubMed: 25129854]

58. Dinh TK, Fendler W, Chalubinska-Fendler J, et al. Circulating miR-29a and miR-150 correlate with delivered dose during thoracic radiation therapy for non-small cell lung cancer. Radiat Oncol. 2016; 11(1) 61-016-0636-4.

59. Sugimachi K, Matsumura T, Hirata $\mathrm{H}$, et al. Identification of a bona fide microRNA biomarker in serum exosomes that predicts hepatocellular carcinoma recurrence after liver transplantation. $\mathrm{Br} \mathbf{J}$ Cancer. 2015; 112(3):532-538. [PubMed: 25584485]

60. Peinado H, Aleckovic M, Lavotshkin S, et al. Melanoma exosomes educate bone marrow progenitor cells toward a pro-metastatic phenotype through MET. Nat Med. 2012; 18(6):883-891. [PubMed: 22635005]

61. Marleau AM, Chen CS, Joyce JA, Tullis RH. Exosome removal as a therapeutic adjuvant in cancer. J Transl Med. 2012; 10 134-5876-10-134.

62. Koga K, Matsumoto K, Akiyoshi T, et al. Purification, characterization and biological significance of tumor-derived exosomes. Anticancer Res. 2005; 25(6A):3703-3707. [PubMed: 16302729] 
63. Ciravolo V, Huber V, Ghedini GC, et al. Potential role of HER2-overexpressing exosomes in countering trastuzumab-based therapy. J Cell Physiol. 2012; 227(2):658-667. [PubMed: 21465472]

64. Yang T, Martin P, Fogarty B, et al. Exosome delivered anticancer drugs across the blood-brain barrier for brain cancer therapy in danio rerio. Pharm Res. 2015; 32(6):2003-2014. [PubMed: 25609010]

65. EL Andaloussi S, Mager I, Breakefield XO, Wood MJ. Extracellular vesicles: Biology and emerging therapeutic opportunities. Nat Rev Drug Discov. 2013; 12(5):347-357. [PubMed: 23584393]

66. Pitt JM, Andre F, Amigorena S, et al. Dendritic cell-derived exosomes for cancer therapy. J Clin Invest. 2016; 126(4):1224-1232. [PubMed: 27035813]

67. Batrakova EV, Kim MS. Using exosomes, naturally-equipped nanocarriers, for drug delivery. J Control Release. 2015; 219:396-405. [PubMed: 26241750]

68. Ohno S, Takanashi M, Sudo K, et al. Systemically injected exosomes targeted to EGFR deliver antitumor microRNA to breast cancer cells. Mol Ther. 2013; 21(1):185-191. [PubMed: 23032975]

69. Ono S, Lam S, Nagahara M, Hoon DS. Circulating microRNA biomarkers as liquid biopsy for cancer patients: Pros and cons of current assays. J Clin Med. 2015; 4(10):1890-1907. [PubMed: 26512704]

70. Greening DW, Xu R, Ji H, Tauro BJ, Simpson RJ. A protocol for exosome isolation and characterization: Evaluation of ultracentrifugation, density-gradient separation, and immunoaffinity capture methods. Methods Mol Biol. 2015; 1295:179-209. [PubMed: 25820723]

71. Ji H, Chen M, Greening DW, et al. Deep sequencing of RNA from three different extracellular vesicle (EV) subtypes released from the human LIM1863 colon cancer cell line uncovers distinct miRNA-enrichment signatures. PLoS One. 2014; 9(10):e110314. [PubMed: 25330373]

72. Colombo M, Moita C, van Niel G, et al. Analysis of ESCRT functions in exosome biogenesis, composition and secretion highlights the heterogeneity of extracellular vesicles. J Cell Sci. 2013; 126(Pt 24):5553-5565. [PubMed: 24105262]

73. Szatanek R, Baran J, Siedlar M, Baj-Krzyworzeka M. Isolation of extracellular vesicles: Determining the correct approach (review). Int J Mol Med. 2015; 36(1):11-17. [PubMed: 25902369]

74. Taylor DD, Shah S. Methods of isolating extracellular vesicles impact down-stream analyses of their cargoes. Methods. 2015; 87:3-10. [PubMed: 25766927] 


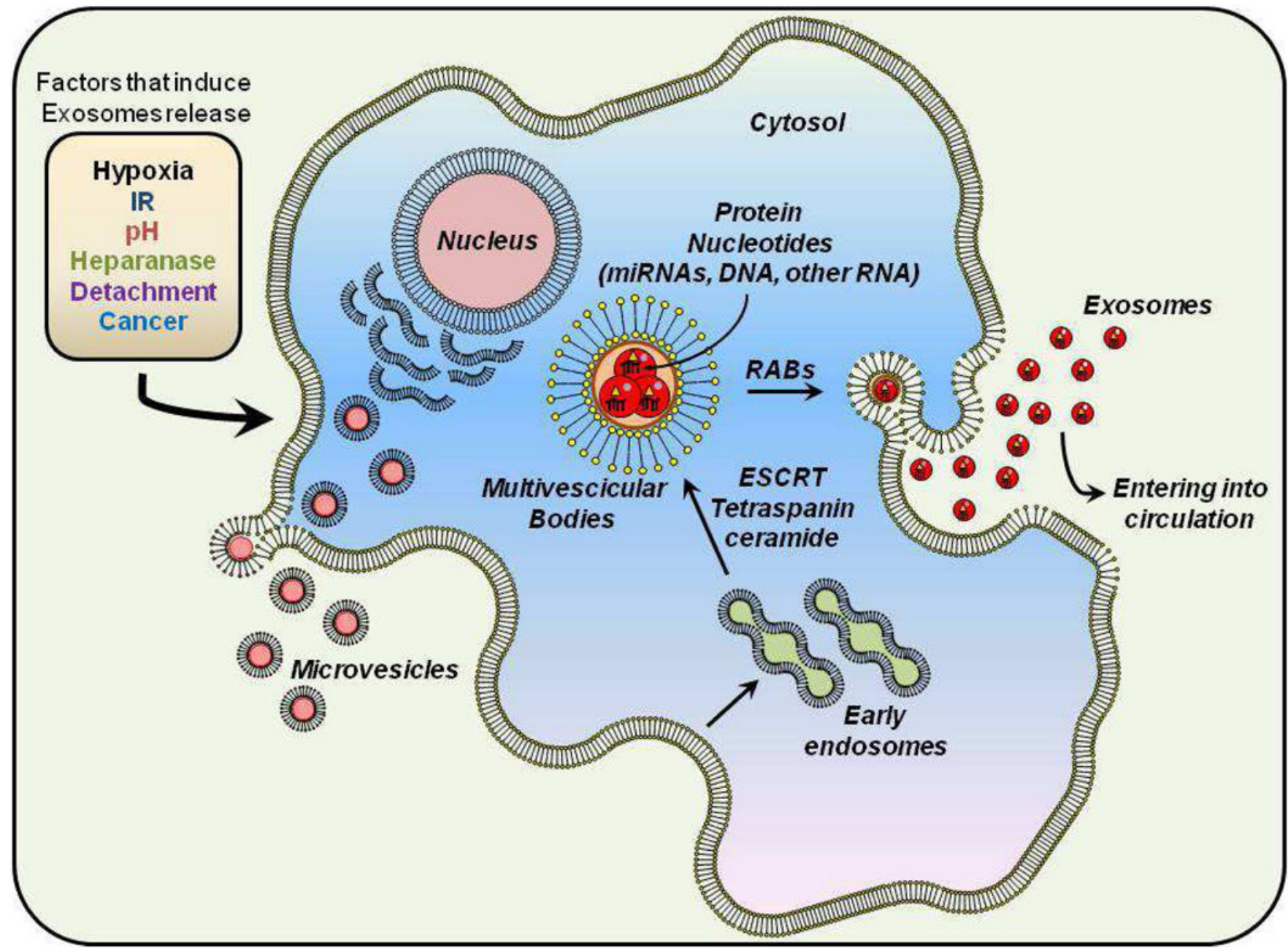

Figure 1. Extracellular Vesicle Biogenesis and secretion

Extracellular vesicles (EVs) form from invagination of the early endosome capturing content including nucleotides and proteins. This content reflects the physiological and pathological state of the cell. The EVs fuse with the plasma membrane and are released into the extracellular space. EVs have been identified in biological fluids including the blood and urine. 
Table 1

Diagnostic, prognostic and predictive extracellular vesicle miRNAs in cancer.

\begin{tabular}{|l|l|l|l|}
\hline miRNAs & Clinical use & Tumor type & reference \\
\hline Diagnosis & & & \\
\hline miR-17-5p, miR-21 & benign vs malignant & pancreatic & Que 2013 \\
\hline miR-200a, 200b, 200c & benign vs malignant & ovarian & Meng2016 \\
\hline miR-21 & HCC vs cirrhosis and Hepatitis B infection & HCC & Wang 2014 \\
\hline miR18a, miR-221, miR-2222, miR-224 & HCC vs cirrhosis & HCC & Sohn 2015 \\
\hline Prognosis/Predictive & & & \\
\hline miR-200b and miR-200c & overall survival & ovarian & Meng 2016 \\
\hline miR-17, miR-19a, miR-21, miR-126, miR-149 & metastic disease & melanoma & Pfeffer2015 \\
\hline miR-19a & overall survival, disease free survival & colorectal & Matsumura 2015 \\
\hline miR-1290, miR-375 & castrate-resistant & prostate & Huang 2015 \\
\hline miR-29a, miR-150 & radiation toxicity & NSCLC & Dinh 2016 \\
\hline miR-718 & recurrence & HCC & Sugimachi 2015 \\
\hline
\end{tabular}


Table 2

Clinical trials evaluating extracellular vesicle in cancer.

\begin{tabular}{|c|c|c|c|}
\hline Name & Sponsor & Status & ID \\
\hline $\begin{array}{l}\text { Pilot Study With the Aim to Quantigy a Stress Protein in the } \\
\text { Blood and in the Urine for the Monitoring and Early Diagnosis } \\
\text { of Malignant Solid Tumors (EXODIAG) }\end{array}$ & Centre Georges Francois Leclerc & recruiting 2016 & NCT02662621 \\
\hline $\begin{array}{l}\text { Circulating Exosomes as Potential Prognostic and Predictive } \\
\text { Biomarkers in Advanced Gastric Cancer Patients (EXO-PPP } \\
\text { Study) }\end{array}$ & Hospital Miguel Servet & recruiting 2015 & NCT01779583 \\
\hline $\begin{array}{l}\text { A Pilot Study of Tumor-Derived Exosomes as Diagnostic and } \\
\text { Prognostic in Breast Cancer patients Receiving Neoadjuvant } \\
\text { Chemotherapy }\end{array}$ & Leo W. Jenkins Cancer Center & Withdrawn & NCT01344109 \\
\hline $\begin{array}{l}\text { Clinical Validation of a Urinary Exosome Signature in Men } \\
\text { Presenting for Suspicion of prostate Cancer }\end{array}$ & Exosome Diagnostics, inc. & completed & NCT02702856 \\
\hline $\begin{array}{l}\text { Exosome Testing as a Screening Modality for Human } \\
\text { Papillomavirus-Positive Oropharyngeal Squamous Cell } \\
\text { Carcinoma }\end{array}$ & New Mexico Cancer Care Alliance & recruiting 2016 & NCT02147418 \\
\hline $\begin{array}{l}\text { Interrogation of Exosome-mediated Intercellular Signaling in } \\
\text { Patients with Pancreatic Cancer }\end{array}$ & $\begin{array}{l}\text { Memorial Sloan Kettering Cancer } \\
\text { Center }\end{array}$ & recruiting 2016 & NCT02393703 \\
\hline $\begin{array}{l}\text { Edible Plant Exosome Ability to Prevent Oral Mucositis } \\
\text { Associated with Chemoradiation of Head and Neck Cancer }\end{array}$ & James Graham Brown Cancer Center & recruiting 2016 & NCT01668849 \\
\hline Blood Collection from People With Ovarian Cancer & National Cancer Institute & recruiting 2015 & NCT02063464 \\
\hline $\begin{array}{l}\text { Early Biomarkers of Tumor Response in High Dose } \\
\text { Hypofractioned Radiotherapy Word package 3: Immune } \\
\text { Response }\end{array}$ & Centre Oscar Lambret & recruiting 2015 & NCT02439008 \\
\hline $\begin{array}{l}\text { Prediction of Response to Kinase Inhibitors Based on Protein } \\
\text { Phosphorylation Profiles in Tumor Tissue from Advanced } \\
\text { Renal Cell Cancer Patients }\end{array}$ & H.M.W. Verheul & recruiting 2015 & NCT02071719 \\
\hline $\begin{array}{l}\text { Study of Molecular Mechanisms Implicated in the } \\
\text { Pathogenesis of Melanoma. Role of Exosomes }\end{array}$ & $\begin{array}{l}\text { Centre Hospitaller Universitaire de } \\
\text { Nice }\end{array}$ & recruiting 2016 & NCT02310451 \\
\hline $\begin{array}{l}\text { Antisense 102: Pilot Immunotherapy for Newly Diagnosed } \\
\text { Malignant Glioma }\end{array}$ & Thomas Jefferson University & recruiting 2016 & NCT02507583 \\
\hline $\begin{array}{l}\text { Evaluation of MicroRNA Expression in Blood and Cytology } \\
\text { for Detecting Barret's Esophagus and Associated Neoplasia }\end{array}$ & $\begin{array}{l}\text { Midwest Biomedical Research } \\
\text { Foundation }\end{array}$ & recruiting 2015 & NCT02464930 \\
\hline Pilot Immunotherapy Trial for Recurrent malignant Gliomas & Thomas Jefferson University & completed & NCT01550523 \\
\hline $\begin{array}{l}\text { Molecular Studies and Clinical Correlations in Human } \\
\text { Prostatic Disease }\end{array}$ & $\begin{array}{l}\text { Memorial Sloan Kettering Cancer } \\
\text { Center }\end{array}$ & recruiting 2016 & NCT00578240 \\
\hline $\begin{array}{l}\text { Development of a Nutrigenetic Test for Personalized } \\
\text { Prescription of Body Weight Loss Diets (Obekit) }\end{array}$ & $\begin{array}{l}\text { Clinica Universidad de Navarra, } \\
\text { Universidad de Navarra }\end{array}$ & recruiting 2015 & NCT02737267 \\
\hline $\begin{array}{l}\text { Pathogenic Mechanisms of Port Wine Stain and Repository of } \\
\text { Port Wine Stain Biopsy Samples }\end{array}$ & University of California, Irvine & recruiting 2016 & NCT02051101 \\
\hline
\end{tabular}

